A. Pramesh Rao, G. Swarup and Gopal-Krishna, eds.

\title{
Geometric Phase in Phasing of Antenna Arrays
}

\author{
Rajendra Bhandari \\ Raman Research Institute, Bangalore-560 080, India
}

\begin{abstract}
The response of a pair of differently polarized antennas is determined by their polarization states and a phase between them which has a geometric part which becomes discontinuous at singular points in the parameter space. Some consequences are described.
\end{abstract}

Introduction The geometric phase (also popularly known as Berry's phase) finds some of its most easily visualizable manifestations in the physics of polarized light (Bhandari 1997). In 1956, Pancharatnam defined the 'in-phase' condition for two different, non-orthognal polarization states to be one for which their interference yields maximum intensity and discovered that under a cycle of transformations of the polarization state along a closed geodesic polygon on the Poincare sphere (PS) the beam acquires a phase equal to half the solid angle subtended by the polygon at the centre. Further work by the present author has shown that the above geometric phase exhibits measurable jumps at singular points in the parameter space such that a circuit around the singularity results in a measurable phase shift equal to $2 n \pi$ (Bhandari 1991, 1997). The flat behaviour of the phase near a singularity has been used in adaptive optics to make a spatial light modulator for pure intensity modulation, keeping phase constant (Gordon and Gourlay 1996). In arrays phased by geometric phase shifters (Fox 1947), phase singularities lead to the possibility of an array looking in two different directions at two different wavelengths (Bhandari 1995).

A pair of antennas with different polarization Take two identical elliptically polarized antennas, in phase with each other, so that their resultant intensity response is maximum 'on-axis'. Now rotate one of the antennas with respect to the other by an angle $\phi / 2$ (figure 1). The two will no longer be in phase in that their combined response will not be maximum 'on-axis'. The phase difference $\psi$ between them (Pancharatnam), given by $\tan \psi=\cos \theta \tan (\phi / 2)$, is shown in figure 1 for a few values of the polar angle $\theta$ of the states on the PS. Note the phase shift (i) is of magnitude $\pi$ for a $2 \pi$ rotation on the PS and (ii) jumps through $\pm \pi$ near $\theta=90^{\circ}, \phi=180^{\circ}$ (as happens for $2 \pi$ rotation in real space of particles with odd half integer spin, verified in analogous polarization experiments in an optical interferometer (Bhandari 1993)). We note that a similar behaviour is implicit in the special case $\theta_{1}=\theta_{2}, \mathrm{Q}=\mathrm{V}=\mathrm{U}=0$ of equation (8) in Morris, Radhakrishnan, \& Seielstad (1964) ${ }^{2}$

\footnotetext{
${ }^{2}$ This equation has been re-derived by Weiler (1973) and Nityananda (1994).
} 

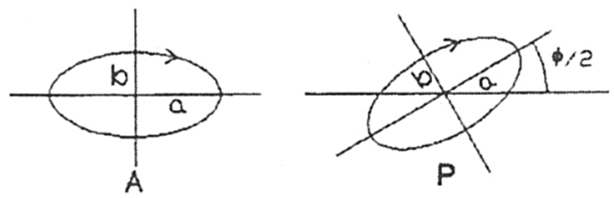

$0 / 6 \tan \left(45^{\circ}-\theta / 2\right)$
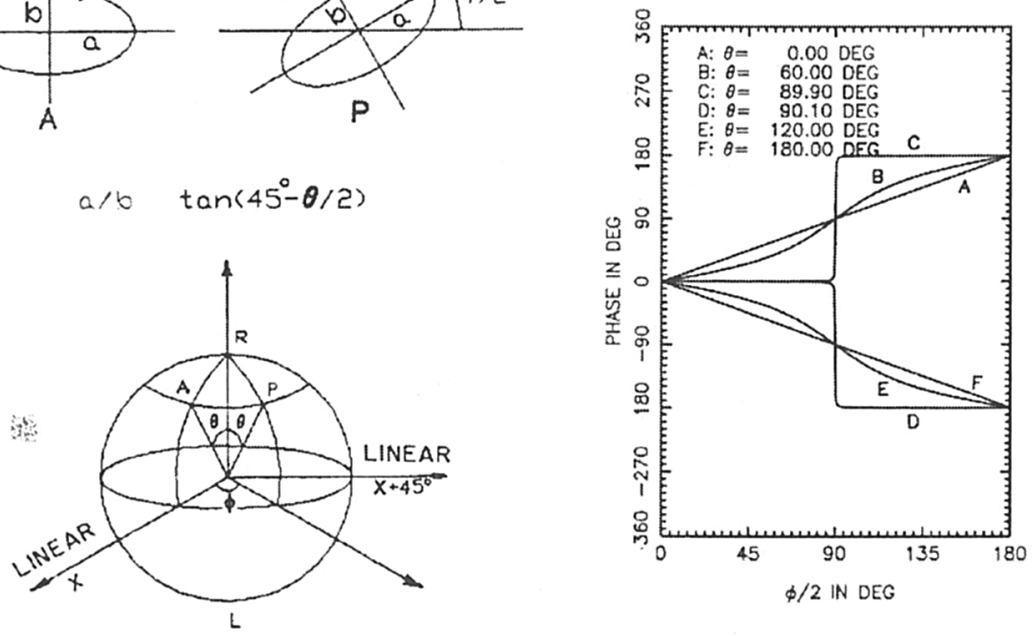

Figure 1. Two elliptically polarized antennas in states A and P, in phase when $\phi=0$, get out of phase when $\phi \neq 0$, by an amount shown in the curves on the right.

Interference nulls for non orthogonal states When radiation from a partially polarized source with degree of polarization $\mathrm{p}$ and eigenpolarizations $P$ and $\tilde{P}$ is picked up by two antennas tuned to polarizations $A_{1}$ and $A_{2}$, then for every polarization state $A_{1}$, there is a state $A_{2}$, not orthogonal to $A_{1}$, such that the correlation of the two outputs is zero (Radhakrishnan 1994). A simple way to prove this curious result is to consider a superposition of two interference patterns; (i) due to a fraction $(1+p) / 2$ of the radiation in state $P$ and (ii) due to a fraction $(1-p) / 2$ in state $\tilde{P}$, with a phase difference of magnitude $\pi$, which is a geometric phase due to the surface enclosed by the closed geodesic curve $P A_{1} \tilde{P} A_{2} P$ on the PS (a hemisphere).

\section{References}

Bhandari R. 1997, Phys. Rep., 281, 1

Bhandari R. 1991, Phys. Lett. A, 157, 221

Love G. D., Gourlay J. 1996, Opt. Lett., 21, 1496

Fox A. G. 1947, Proc. I.R.E., 35, 1489

Bhandari R. 1995, Phys. Lett. A, 204, 188

Bhandari R. 1993, Phys. Lett. A, 180, 15

Morris D., Radhakrishnan V., \& Seielstad G. A. 1964, Ap.J., 139, 551

Weiler K. W. 1973, Astron. \& Astrophys., 26, 403

Nityananda R. 1994, Current Science, 67, 243

Radhakrishnan V. 1994, Current Science, 67, 257 